\title{
Gegen Schlegels Meinung über die Verfärbung des Gefieders.
}

\author{
Von

\section{Pastor Chr. L. Brehm.}

Im Anschlusse an das Vorstehende halte ich es für nothwendig, noch Einiges über $S$ chlegels gesammte Verfärbungstheorie zu bemerken. *) Die Grundzüge derselben sind folgende:

„Die jungen Vögel mausern zum ersten Male im Herbste desjenigen Jahres, welches auf das folgt, in welchem sie ausgebrütet worden sind."

„Die Mauser findet stets nach der Bruiezeit, wenn die Jungen selbständig geworden sind, Statt."

"Ausser der Mauser beobachtet man aber bei den meisten Vögeln noch: a) eine Erneuerung-kleiner, früher verloren gegangener Federn; b) das Entstehen neuer Federn, welche nicht bestimmt sind, fruher dagewesene zu ersetzen: z. B. der Federn des Halskragens bei Tringa pugnax, der langen Federn der Paradiesvögel, u s. w "

„Mit der Entwickelung des Geschlechtstriebes werden zugleich durch neu beginnenden Zufluss von Saften die Federn, deren Gefässe anscheinend vertrocknet waren, nochmals befähigt, den äusseren Einflüssen durch eine innere Kraft zu widerstehen und sich nach einem längeren oder kürzeren Stillstande aufs Neue auszubilden. Durch diesen Process werden nun die beiden folgenden Haupterscheinungen hervorgebracht:“

„a) Die abgeriebenen oder abgestossenen Federn werden ergänzt durch das Entstehen neuer Barte und Bärtchen. Es werden alsdaun zugleich, wenn sich die Bärte nur bis zu einem gewissen Punkte des Schaftes entwickeln, die nun überflussig gewordenen Spitzen der Federn abgestossen: wesshalb die Feder in diesem Falle kürzer wird; oder die Federn nehmen, wie es bei manchen Vögel der Fall ist, eine von ihrer früheren verschiedene, meist verlängerte Gestalt an. ${ }^{\alpha}$

„b) Zu dieser Zeit tritt auch eine grössere Menge Pigment in die Federn, wie diess auch in dem Schnabel, den Fussen und dem nachsten Theile der Haut Statt findet."

„Durch diesen Prốcess, und nícht durch die Mauser, entsteht das vollkommene Prachtkleid der meisten Vỏgel. Dieses Prachtkleid wird nur durch den Wechsel und das Entstehen einzelner neuer Federn vervollkommnet."

Diess sind die, allerdings ganz neuen Behauptungen, welche Herr Schleg el vorträgt, welche neuerlich aber schon Herr von Home y er in dem 1. Hefte der "Naumannia," Jahrgang 1853, S. 64-78, geprüt und grössten Theils wide rlegt hat.

Schlegels Name hat jedoch einen so guten Klang, und zugleich ist die Sache selbst so wichtig und die Zahl ihrer Anhänger so gross, ") dass ich es nicht für überflüssig halte, in dieser Zeitschrift auch meine Ansicht über die Frage zu äussern.

Die Behauptung $\mathbf{S} \mathrm{chleg} e \mathrm{~s}$, dass ein junger Vogel erst im 2. Herbste seines Lebens zum ersten Male sich mausern solle, ist eine

") Siehe „Naumannia," Jahrg. 1852, Heft II, S. $19-40$.

") Eben dieser Anklang, welchen sie mehrseitig, wemn auch nur innerhalb sehr viel beschränkterer, daher erst zu ermittelnder Granzen sofort gefunden hat, zeigt wohl hinlanglich: dass hiermit ein Gegenstand berührt ist, hinsichtlich dessen langst in Vielen das Gefuhl gelegen hat, dass derselbe ein Punki sei, über welchen bisher Niemand ins Klare gekommen war, noch bis hent gekommen ist.

D. Herausg. 
höchst auffallende. Wie es sich bei den Raubvögeln in der Wirklichkeit der Natur mit dieser ersten Mauser verhält, ist bereits in den eben vorangegangenen Aufsätzen ausführlich von mir nachgewiesen. Es bliebe also jetzt noch übrig, in Kürze auch die anderen Vögel in Bezug auf die erste Mauser zu betrachten.

Bei den meisten von ihnen zeigt sofort schon der blosse Augenschein, dass ihr wirkliches, erstes Jugend-Gefieder gar nicht $11 / 4-11 / 2$ Jahr, sondern in der That nur einige Wochen ausdauern $k$ ann. Dahin gehören unter den einheimischen ganz besonders die Fli e g e n fä ng er, Pirole und die eigentlichen Rohrsänger: wie Calamoherpe arundinacea und ihre nächsten Verwandten. Die Federn dieser jungen Vögel sind so zart, weitstrahlig und vergänglich, dass sie sehr bald dauerhafteren und stärkeren Platz machen müssen.- Und diess geschieht auch. Die Jungen der Pirole und Rohrsänger haben kaum das Nest verlassen, so beginnt schon ihre Mauser und bringt ihnen ein neues Gewand: das erste Herbstkleid. So ist es bei allen kleinen Vögeln Ja selbst die grossen Hühner, nämlich die A u er- und B irkh u hner, bekommen im ersten Herbste ihres Lebens ihr ausgefärbtes Kleid; nur bekommen auch sie es nicht durch Ausfärbung des Jugendkleides, sondern durch einen vollständigen Federwechsel.

Manche Vögel tragen nach der ersten Herbstmauser noch das Kleid des Weibchens; so die einheimischen Pirole und Hausrothschwänze. Solche erhalten, wie die meisten Tagraubvögel, die Silberfasane, die Tauchenten, Säger, kleinen Möven und andere, ihr ausgefärbtes Kleid erst im zweiten Lebensjahre; und noch andere, wie viele grosse Raubvögel, die Flamingos, die grossen Möven u.s.w. erst im dritten Lebensjahre: aber stets nur durch Federwechsel.

Arbeitete Herr Schlegel nicht weit mehr in blossen Naturaliensammlungen, als in der freien Natur: dann würden ihn die vielen Männchen der Haussperlinge, der Finken, Grünlinge, Goldammern, $\mathrm{Z}$ eisige und $\mathrm{Hühner}$, die man jeden Winter bei uns findet, eines Anderen und Besseren belehrt haben. Denn diese müssten ja noch das Jugendkleid tragen, anstatt des ausgefärbten, welches sie wirklich haben. Dass ausser der Mauser an Stellen, wo keine besonders auffallende Federn gestanden haben, neue dergle chen hervorwachsen sollen, wie z. B. die langen Halsfedern des $K a m p f h a h n-M a ̈ n n-$ chens, bezweifle ich mit Herrn von Homeyer ganz. *)

Bei den Männchen des Machetes pugnax mussen wenigstens erst die Federn des Herbstkleides ausfallen, ehe die langen der Krause hervorwachsen können. Was nun die Ausbildung alter, schon lange gestandener Federn zur Paarungszeit anlangt, - nämlich, dass sie, abgenutzt und

*) Ganz dasselbe, gerade in Bezug auf Machetes pugnux, hat bekanntlich auch schon Gloger xum Voraus in seinem ersten Aufsatze (Heft I d. Journ., S. 22 u. 23 , Note) gethan: als der Schlegelsehe Artikel noch gar nicht gedruckt war; ebenso Martin in seinem zweiten, Hef 111, S. 211. Diese, wie das von Schlegel behauptete Wegfallen der Mauser junger Vögel in dem ersten Herbste ihres Lebens, und mehreres Andere, war es ja uherhaupt, worin unsere gesammten erfahrueren Praktiker sofort überall gegen Schl. einig waren. D. Herausg. 
abgerieben, sich durch Hervorwachsen neuer Bärte oder Bärtchen wieder ergänzen, oder, (wie bei den Silberreihern, manchen EntenIännchen und mehreren anderen Vögeln,) sich verlängern und so eine andere Gestalt annehmen können: -- so beruht diese Meinung auf einem sehr grossen Irrthume, welcher sich überhaupt durch S c h l e g e ls gauze Theorie hinzieht und somit eine grosse Menge ganz unrichtiger Folgerungen herbeigeführt hat. Herr Schlegel sagt ja selbst: dass eine Vogelfeder mit dem Blatte eines Baumes Aehnlichkeit habe; ein Vergleich, in welchem ich ihm ganz beistimme. Hätte er denselben doch nur auch festgehalten! Dann würde er geschlossen haben: dass eine Feder, wenn sie nach ihrer völligen Ausbildung eine Zeit lang gestanden hat, eben so wenig wie das Blatt einen neuen Anlauf zum Wachsen nehmen und eine verlängerte, oder sonst vollkommnere Gestalt annehmen kann. Dass also z. B. eine, an den Seiten sägezackenförmig-abgenutzte Schulter- oder hintere Schwungfeder eines Sumpfvogels sich wieder ergänzen und sonach mit ungezähnelten Seiten, (an welchen die Bärte nachgewachsen seien,) erscheinen kann, ist eine physische Unmöglichkeit. Dass Schlegel diese Federn bei den in Holland brütenden Sumpf-, Strand-, Wasser-, Schlamm- und U ferläufern etc. vollkommen ergänzt gesehen hat, bezweifeln wir, Herr von Homeyer, und ich, keinesweges; denn auch wir haben diess ja sehr oft beobachtet. Nur waren eben diese Federn nicht nach-, sondern frisch hervorgewachsen: wie bei den Piepern, Schaf- und Bachstelzen, sammt manchen anderen. So verhält es sich auch mit den ausgefärbten Federn der jungen Tauchenten: $z$. B. mit denen, welche Herr Martin im III. Hefte d. „Journ." bei einer, im März dieses Jahres erlegten einjährigen Trauerente, Anas nigra Linn., fand. Allerdings bemerkte er, so wie die anderen mituntersuchenden Ornithologen, weder äusserlich, noch auf der inneren Seite der abgezogenen Haut eine Spur von Kielen, . also von Federwechsel; jedoch nur aus dem einfachen Grunde, weil diese Federn schon im Winter hervorgewachsen waren und so ihre vollkommene Ausbildung schon erhalten hatten, ehe die Ente getödtet wurde. Aber der Schluss, dass diese neuen Federn alte, in ausgefärbte umgewandelte wären, hätte schon aus dem Grunde nicht aus der Erscheinung gezogen werden sollen, weil die andere Trauerente, von deren schwarzen Federn einige wenige noch Kiele hatten, also frisch hervorgewachsen waren, die Erklärung des Răthsels an die Hand gab. Warum sollen denn diese neu hervor-

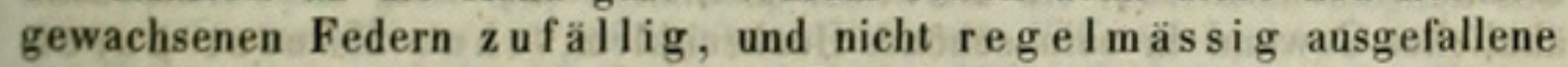
ersetzt haben? Die an der anderen Ente beobachtefen konnten ja diess eben so gut sein, wie die mit den Kielen. Die Sache ist einfach die: Bei der einen Trauerente war diese erste Mauser, welche ungemein langsam von Statten geht, - denn sie dauert 8 bis 9 Monate, eine kurze Zeit unterbrochen; bei den anderen hingegen dauerte sie fort. Eben desshalb fand Herr Martin bei der einen schwarze Federn ohn e blutige Kiele, bei der anderen $\mathrm{m}$ it solchen Kielen.

Die Meinung, dass alte Federn sich in neue von ganz anderer Gestalt umwandeln könnten, verleitet Herrn Schlegel zu sehr sonderbaren Behauptungen. 
So sollen die Männchen der wilden $\mathrm{Hühner,} \mathrm{des} \mathrm{Gallus} \mathrm{bankiva,}$ G. furcatus etc., durch blosse Ausbildung der Federn des Jugendkleides ihr Prachtkleid erhalten: eine Behauptung, welche ein Jeder widerlegen muss, der je selbst Haushühner gehabt hat. Denn die wilden Gallus bankiva können ihre schönen Federn doch gewiss auch nicht auf andere Weise bekommen, als die zahmen. Ich habe aber gesehen, dass das Prachtkleid des Gallus bankiva die dritte Befiederung ist, welche der Vogel erhält: weil das eigentliche Jugendkleid, also dasjenige, welches sich nach Schlegel ohne Mauser in das Prachtkleid verwandeltn soll, schon die Stelle der ersten Befiederung, (welche das kleine Küchelchen trägt und nach und nach beim Grösserwerden verliert,) eingenommen hat. -

Wie es mit dieser, von Schlegel behaupteten Umwandelung der alten Federn in neue anders gestaltete steht: nicht viel besser sieht es mit seiner Verfärbungs- oder vielmehr Ausfärbungstheorie in das Prachtkleid, welches nach ihm stets das hochzeitliche sein soll, aus.

Es ist schon uuwahr, dass alle Vögel zur Paarungszeit am Schönsten gefärbt sein sollen. Diess ist z. B. nicht der Fall bei den Raubvögeln, den krähen- und spechtartigen, den Schwarzamseln, bei mehren Drosseln und Sängern: (so namentlich bei den schwarzköpfigen und Klapper-Grasmücken, den Rothkehlchen;) ferner nicht bei den Scharben, Pelekanen und vielen anderen.

Aber nicht ein einziger Vogel geht aus dem Jugendkleide durch blosseVerfärbung in das ausgefärbte úber.

Ich läugne zwar nicht, dass manche Vögel zur Brutzeit eine höhere Färbung bekommen; dahin gehören unter den deutschen besonders die Bluthänflinge, die Leinzeisige, die Edelfinken und die Teichhühner, Stagnicola Brhm.; (Gallinula chloropus Lath.) Allein die Bluthänflingmännchen erreichen ihre grösste Schönheit nicht zur Paarungszeit, sondern erst nach derselben, und zwar im Juli; und bei den Teichhühnern sind es nur die einjährigen Männchen, welche sich ausfärben, d. h. zur Brutzeit schöner werden. Auch die männlichen B la u k ehl ch en zeigen zur Brutzeit das schönste Blau. Aber die Behauptung Schlegels: dass eine Lamprotornis und ein Euplectes aus dem unscheinbaren bräunlichen Kleide in das Prachtkleid ubergingen; *) dass ein Schneehuhn sein weisses Winterkleid in das braune Sommerkleid, ein Entenmännchen sein, dem des Weibchens ähnliches Gewand in sein schönes Hochzeitkleid, ohne Mauser durch blosses A usfärben verwandeln solle, - beruht auf einem gros-

*) Ein hiesiger Natur - und besonders Vogelfreund, - der sorgsamste Pfleger und Beobachter von Stubenthieren, den es nur geben kann, - Hr. Hiseler, unterhält seit beinahe 2 Jahrzehenten stets finkenartige Vögel aus der Zahl der mit Euplectes nahe verwandten Gattungen, zum Theil auch von jenen selbst. $\mathbf{E r}$ hat auf diese Weise fast alle, nur irgend zu erlangende Species von $\mathrm{S}$ a $\mathbf{m} \mathbf{m} \mathbf{t}$ - und Feuerfinken, Whidafinken etc., entweder früher besessen, oder besitzt sie noch heut. 'Niemals hat er jedoch das Prachtgefieder jener, oder die langen Schwanzfedern dieser, mit dem Wechsel der Jahreszeit anders zum Vorscheine kommen geselıen, als: durch wirkliches Mausern. Keine Spur vou Umfärbung, oder 
sen Irrthume. Wir können den wirklichen Uebergang dieser Kleider durch Federwechsel deutlich nachweisen.

Nur bei den Seeschwalben bin ich über die Verfärbung des Herbstkleides in das Hochzeitkleid ungewiss, und enthalte mich desswegen jedes Urtheils über dieselben. Bei den anderen Vögeln hingegen, die ich habe untersuchen können, weiss ich gewiss: dass eine solche Verfärbung nicht Statt findet; sondern dass die Veränderung der Kleider nur durch Mauser bewirkt wird.

Da ich nun bei den bekanntesten deutschen Vögeln, namentlich bei den Hausperlingen, Finken, Stockenten etc., die Annahmen Schlegels nicht bestätigt gefunden habe: so muss es mir erlaubt sein, auch in die anderen, die ausländischen Vögel betreffenden Behauptungen unseres Freundes einigen Zweifel zu setzen. Ebenso bin ich fest überzeugt, dass das von Herrn Martin behauptete allmähliche Schwarzwerden der Fli egenfängermännchen auf einem Irrthume beruht: weil ich solche Vögel besitze, welche im Juli geschossen sind und gleichwohl nur halb-schwarze Federn zeigen. Wäre die allmähliche Verfärbung dieser Vögel richtig: dann müssten die im Juli erlegten doch endlich ihre Ausbildung erlangt haben. Ich halte diese für einjährige, die, wie Hausrothschwänze, ihr ausgefärbtes Kleid noch nicht angelegt haben, daher nicht rein schwarz aussehen. Die doppelte Mauser der Fligenfänger, d. h. der s chwarzrü ckigen und $\mathrm{Hal}$ sbandfli e genfänger, (denn die kleinen und rothhälsigen haben nur einen einfachen Federwechsel,) erkennt man am deutlichsten an den 3 hintersten Schwungfedern, welche ganz anders gestaltet und gezeichnet sind, als bei den Herbstvögeln.

So wenig wir beide, Herr von Homeyer und ich, mit Schlegels Ansichten übereinstimmen: so gern verdanken wir ihm dennoch seine Bekanntmachung derselben. *) $\mathrm{Er}$ hat dadurch einen Gegenstand zur Sprache gebracht, welcher von grosser Wichtigkeit ist, und hat somit eine bestimmtere Erörterung desselben veranlasst, welche der Wissenschaft mittelbar, wie unmittelbar nur förderlich sein kann.

gar von Umgestaltung des lerchenartig-bräunlichen Gefieders nach seiner Structur, ohne Mauser! - Er wird übrigens wohl auch nächstens Einiges hierüber, wie über sonst dahin Gehöriges, für unsere Zeitschrift einliefern.

D. Herausg.

*) Die Standpunkte, welche die Herren Brehm und v. Homeyer zu der Frage einnehmen, bleiben aber doch wohl noch sehr wesentlich verschiedene. Hr. v. Hom e yer giebt eine Verfarbung theilweise als recht wohl möglich und sogar wahrscheinlich zu; er bekämpft nur Schlegels Uebertreibungen, und führt das Wahre daran auf das bereits von Anderen früher Geahnte zurück. Er befindet sich also hiermit im Ganzen bloss in demselben Falle, wie Martin und wie besonders Gloger. Hr. Brehm dagegen bestreitet offenbar jede Verfärbung. Die Sache bleibt sonach, lis sub judice,. und wird es vermuthlich theilweise noch länger bleiben für Alle, wie auch für uns hier (in Berlin). Dagegen bekennen wir uns für solche Fälle, wie der mit Anas nigra, gern als von Hrn. Br. durch Thatsachen richtig belehrt.

D. Herausg. 

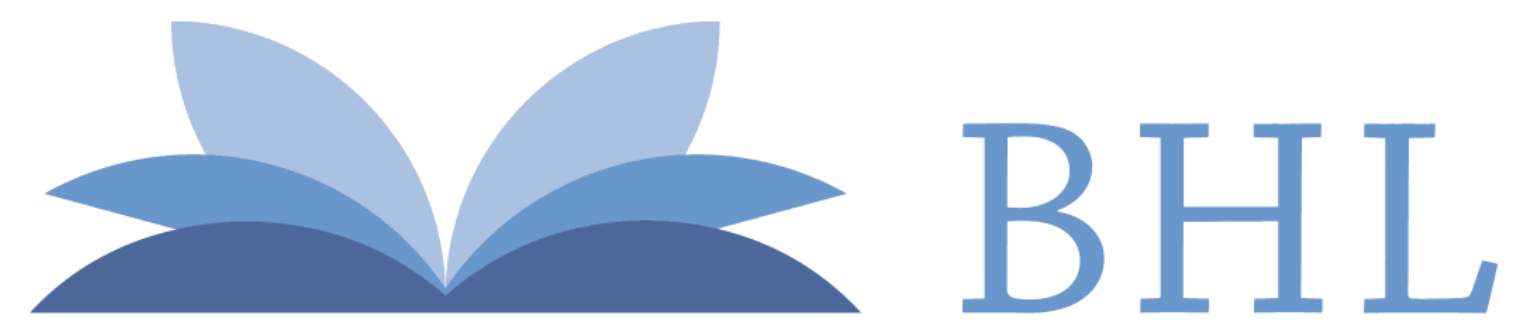

\section{Biodiversity Heritage Library}

1853. "Gegen Schlegels Meinung über die Verfärbung des Gefieders." Journal fu

r Ornithologie 1, 347-351. https://doi.org/10.1007/bf02002562.

View This Item Online: $\underline{\text { https://www.biodiversitylibrary.org/item/49195 }}$

DOI: https://doi.org/10.1007/bf02002562

Permalink: https://www.biodiversitylibrary.org/partpdf/142190

\section{Holding Institution}

Natural History Museum Library, London

\section{Sponsored by}

Natural History Museum Library, London

\section{Copyright \& Reuse}

Copyright Status: Public domain. The BHL considers that this work is no longer under copyright protection.

This document was created from content at the Biodiversity Heritage Library, the world's largest open access digital library for biodiversity literature and archives. Visit BHL at https://www.biodiversitylibrary.org. 